\title{
Bioeffects of albumin-encapsulated microbubbles and real-time myocardial contrast echocardiography in an experimental canine model
}

P.M.M. Dourado ${ }^{1}$,

J.M. Tsutsui' ${ }^{2}$ J.M.T. Santos², V.D. Aiello ${ }^{3}$, W. Mathias Jr. ${ }^{2}$,

J.A.F. Ramires ${ }^{4}$ P.L. da Luz ${ }^{1}$ and A.C.P. Chagas ${ }^{1}$
${ }^{1}$ Laboratório de Investigação em Isquemia Miocárdica, Unidade Clínica de Aterosclerose, ${ }^{2}$ Laboratório de Ecocardiografia, ${ }^{3}$ Laboratório de Patologia, ${ }^{4}$ Divisão Clínica, Instituto do Coração, Faculdade de Medicina, Universidade de São Paulo, São Paulo, SP, Brasil

\section{Correspondence}

P.M.M. Dourado

Laboratório de Investigação em

Isquemia Miocárdica

Unidade Clínica de Aterosclerose

Instituto do Coração, FM, USP

Av. Dr. Enéas C. Aguiar, 44

05403-900 São Paulo, SP

Brasil

Fax +55-11-3069-5447

E-mail: pmmdourado@terra.com.br

Publication supported by FAPESP. ....................

Received June 17, 2005 Accepted March 3, 2006 .....................

\section{Abstract}

Myocardial contrast echocardiography has been used for assessing myocardial perfusion. Some concerns regarding its safety still remain, mainly regarding the induction of microvascular alterations. We sought to determine the bioeffects of microbubbles and real-time myocardial contrast echocardiography (RTMCE) in a closed-chest canine model. Eighteen mongrel dogs were randomly assigned to two groups. Nine were submitted to continuous intravenous infusion of perfluorocarbon-exposed sonicated dextrose albumin (PESDA) plus continuous imaging using power pulse inversion RTMCE for 180 min, associated with manually deflagrated high-mechanical index impulses. The control group consisted of 3 dogs submitted to continuous imaging using RTMCE without PESDA, 3 dogs received PESDA alone, and 3 dogs were sham-operated. Hemodynamics and cardiac rhythm were monitored continuously. Histological analysis was performed on cardiac and pulmonary tissues. No hemodynamic changes or cardiac arrhythmias were observed in any group. Normal left ventricular ejection fraction and myocardial perfusion were maintained throughout the protocol. Frequency of mild and focal microhemorrhage areas in myocardial and pulmonary tissue was similar in PESDA plus RTMCE and control groups. The percentages of positive microscopical fields in the myocardium were 0.4 and $0.7 \%(\mathrm{P}=\mathrm{NS}$ ) in the PESDA plus RTMCE and control groups, respectively, and in the lungs they were 2.1 and $1.1 \%$, respectively $(\mathrm{P}=\mathrm{NS})$. In this canine model, myocardial perfusion imaging obtained with PESDA and RTMCE was safe, with no alteration in cardiac rhythm or left ventricular function. Mild and focal myocardial and pulmonary microhemorrhages were observed in both groups, and may be attributed to surgical tissue manipulation.
Key words

- Microbubbles

- Ultrasound

- Bioeffects

- Myocardial contrast echocardiography 


\section{Introduction}

Recently, the assessment of myocardial perfusion by echocardiography following intravenous injection of ultrasound contrast agents has proved to be feasible (1). The development of low-mechanical index imaging techniques that cause minimal microbubble destruction has permitted simultaneous analysis of myocardial perfusion and function in real time (2). Real-time myocardial contrast echocardiography (RTMCE) increases the sensitivity of dobutamine stress testing for the detection of coronary artery disease $(3,4)$ and can distinguish between stunned and infarcted myocardium after acute ischemia (5). However, some concerns regarding the safety of this new imaging modality remain, mainly because some animal studies have demonstrated damage to the endothelial system in the presence of microbubbles and ultrasound (6-9). Although investigations of the interaction of ultrasound and contrast agents in vivo are scarce, some effects have been reported in the literature (10) such as capillary damage in organs that contain air such as the lungs (7), damage to the microvasculature (11), and limited capillary ruptures in the heart (12). Moreover, simultaneous exposure of isolated rabbit hearts to ultrasound and a contrast agent has been found to result in transient depression of left ventricular contractile function, an increase in coronary perfusion pressure and in lactate production, and in limited mechanical index-dependent capillary rupture (12). Nevertheless, the clinical importance of these effects has not been established (13).

The bioeffects of the mechanical index during RTMCE imaging are particularly important since one of the current applications of this technique is the determination of myocardial blood flow. The kinetics of microbubbles in the myocardium can be determined by applying a high-mechanical index flash impulse to destroy the microbubbles in the myocardium, followed by measurement of microbubble replenishment velocity and plateau intensity. The application of high-mechanical index pulse sequences may induce microvascular damage (14).

The aim of the present study was to determine the effect of intravenous infusion of albumin-encapsulated microbubbles and RTMCE with intermittent application of high-mechanical index flash impulses on the cardiac and pulmonary microvasculature of dogs.

\section{Material and Methods}

\section{Animal preparation}

Eighteen mongrel dogs were anesthetized intravenously with $30 \mathrm{mg} / \mathrm{kg}$ sodium pentobarbital, intubated and mechanically ventilated with a respirator pump (model 670, Takaoka Medical, São Paulo, SP, Brazil). The anesthesia was supplemented with additional intravenous doses of sodium pentobarbital (2-3 mL) when necessary. A 7-Fr catheter was inserted into the left carotid artery and connected to a Biopac Systems device (model TSD-104, Biopac Systems, Santa Barbara, CA, USA) to record arterial pressure and heart rate. Another 7-Fr catheter was inserted into the femoral vein to infuse drugs and ultrasound contrast and a third catheter was placed into the jugular vein to record central venous pressure. Hemodynamic parameters and electrocardiographic rhythm were monitored continuously. At the end of each experiment, all dogs received an additional dose of $30 \mathrm{mg} /$ $\mathrm{kg}$ sodium pentobarbital before euthanasia.

\section{Myocardial contrast echocardiography}

The contrast agent used in this study consisted of albumin-encapsulated microbubbles (perfluorocarbon-exposed sonicated dextrose albumin, PESDA). Microbubbles were prepared by hand shaking $8 \mathrm{~mL}$ of 
decafluorobutane gas with a 3:1 mixture of $5 \%$ dextrose and 5\% human serum albumin and the mixture was sonicated for $75 \mathrm{~s}$. The mean concentration of microbubbles produced with this technique was $1.4 \times 10^{9}$ microbubbles per $\mathrm{mL}$ and the mean size was $4.6 \pm$ $0.2 \mu \mathrm{m}$ (as reported in Ref. 2). RTMCE was obtained by continuous intravenous infusion of $0.2 \mathrm{~mL} / \mathrm{kg}$ PESDA diluted in $80 \mathrm{~mL}$ of normal saline at a rate of $2-3 \mathrm{~mL} / \mathrm{min}$, injected through the femoral vein.

Echocardiographic images were acquired in a closed-chest model. RTMCE was performed using a commercially available ultrasound system (HDI 5000, Philips Medical Systems, Bothell, WA, USA) equipped with a 4-2 MHz broadband transducer. Imaging was obtained by the power pulse inversion modality at a low-mechanical index (0.1). Specific instrumentation settings were low dynamic range, frame rate $>25 \mathrm{~Hz}$, and maximal line density. After adjustments, these parameters were constant for each experiment. Animals in the groups that received RTMCE were exposed to continuous imaging using power pulse inversion RTMCE for 180 min. A manually triggered, transient, high-mechanical index (1.2) imaging flash was deflagrated at each 12 heartbeats to destroy microbubbles within the myocardium and to permit subsequent myocardial replenishment $(15,16)$. Although the analysis of myocardial perfusion was performed using the apical four-, three-, and two-chamber views, continuous imaging with RTMCE was performed most of the time using the short-axis view at the level of papillary muscles. The images were stored on videotape and on an optical disk for offline processing and analysis.

\section{Study protocol}

The protocol was reviewed and approved by the Institutional Animal Use Committee of the Heart Institute, University of São Paulo Medical School. Experiments were carried out in accordance with the American Heart Association on Research Animal Use (17). The dogs were treated with standard ration and fasted overnight before the experiments.

The effects of ultrasound-induced microbubble destruction were evaluated in two groups. The PESDA plus RTMCE group consisted of 9 dogs weighing $17 \pm 2 \mathrm{~kg}$ which received intravenous albumin-encapsulated microbubbles, PESDA and RTMCE for $180 \mathrm{~min}$, associated with manually deflagrated high-mechanical index flash impulses to destroy the microbubbles every 12 heartbeats. The control group consisted of $9 \mathrm{dogs}$ weighing $17 \pm 2 \mathrm{~kg}$. In this group, $3 \mathrm{dogs}$ received intravenous infusion of PESDA for $180 \mathrm{~min}$ and no ultrasound (PESDA alone), 3 dogs underwent RTMCE imaging for 180 min associated with manually deflagrated high-mechanical index flash impulses without infusion of PESDA (RTMCE alone), and 3 dogs were sham-operated and did not receive PESDA or ultrasound.

Left ventricular systolic function and myocardial perfusion were evaluated at baseline and at 90 and $180 \mathrm{~min}$ during the protocol. Left ventricular ejection fraction was estimated by two-dimensional images (18). The presence of premature ventricular contractions (PVC) or any other cardiac arrhythmias was monitored continuously with a one-lead electrocardiogram connected to the echocardiographic system. Histological analysis of lung and heart samples was performed at the end of the protocol.

\section{Histological analysis}

At the end of each experiment, the animals were euthanized with an intravenous injection of potassium chloride and the heart and lungs were excised. The heart was cut transversally from the apex to the base and transmural fragments $(1.5 \times 1.0 \mathrm{~cm})$ of septal, anterior, and lateral walls were excised at the level of the papillary muscles for histological analysis. Two fragments of the left 
lung $(2.0 \times 1.0 \mathrm{~cm})$, one from the hilar region and the other sub-pleural, from the apex of the cranial lobe, were also examined. After fixation in $10 \%$ buffered formalin, the fragments were submitted to standard histological processing. Five-micrometer thick sections were obtained, stained with hematoxylin-eosin and examined semi-quantitatively under the light microscope. The mean numbers of 100X microscopical fields evaluated were 100 for the lung fragments and 120 for the hearts. We searched for areas of necrosis, contraction bands, and hemorrhage. The percentage of "positive" microscopic fields relative to the total number of analyzed fields was determined.

\section{Statistical analysis}

Continuous and normally distributed data are reported as mean \pm one standard deviation (SD) and qualitative data are reported as proportions. Comparisons of hemodynamic data and left ventricular ejection fraction at baseline and at 90 and 180 min of treatment were performed by repeated-measures analysis of variance or the Friedman test, as appropriate. Inter- and intra-group comparisons of continuous variables were performed by the unpaired and paired Student $t$-test, respectively. Comparison of proportions was performed by the exact Fisher test. A P value

\begin{tabular}{|c|c|c|c|c|}
\hline \multirow[t]{2}{*}{ Histological findings } & \multirow{2}{*}{$\begin{array}{c}\text { PESDA + RTMCE } \\
(\mathrm{N}=8)\end{array}$} & \multicolumn{3}{|c|}{ Control group $(\mathrm{N}=8)$} \\
\hline & & $\begin{array}{l}\text { PESDA } \\
\text { alone }\end{array}$ & $\begin{array}{l}\text { RTMCE } \\
\text { alone }\end{array}$ & $\begin{array}{l}\text { Sham- } \\
\text { operated }\end{array}$ \\
\hline \multicolumn{5}{|l|}{ Myocardium } \\
\hline Focal hemorrhage & 2 & 1 & 2 & 1 \\
\hline Contraction bands & 0 & 0 & 0 & 1 \\
\hline \multicolumn{5}{|l|}{ Lung } \\
\hline Focal alveolar hemorrhage & 3 & 0 & 1 & 2 \\
\hline Perivascular focal hemorrhage & 2 & 0 & 0 & 2 \\
\hline
\end{tabular}

Data are reported as number of dogs.
$<0.05$ (two-sided) was considered to be statistically significant.

\section{Results}

Intravenous infusion of PESDA microbubbles permitted the analysis of myocardial perfusion and left ventricular function in all dogs during the protocol. Two dogs were excluded, one from the PESDA plus RTMCE group and the other from the PESDA group because of hemodynamic instability before the beginning of microbubble infusion, which was attributed to the effects of anesthesia and hypovolemia. Baseline left ventricular ejection fraction was normal in all dogs with no difference between PESDA plus RTMCE and control groups $(0.70 \pm$ 0.03 vs $0.69 \pm 0.04 ; \mathrm{P}=0.74)$. No changes in left ventricular ejection fraction were observed at 90 or $180 \mathrm{~min}$ of the protocol in the PESDA plus RTMCE group $(\mathrm{P}=0.8)$ and in the control group $(\mathrm{P}=0.56)$. Myocardial perfusion was normal in all animals.

At baseline, no differences were observed in heart rate $(128.6 \pm 23.8$ vs $128.8 \pm 23.9$ bpm; $\mathrm{P}=0.98$ ), mean arterial blood pressure $(93.1 \pm 9.9$ vs $91.3 \pm 16.2 \mathrm{mmHg} ; \mathrm{P}=0.69)$ and central venous pressure (1.6 \pm 1.5 vs 1.5 $\pm 2.0 \mathrm{cmH}_{2} \mathrm{O} ; \mathrm{P}=0.52$ ) between PESDA plus RTMCE and control groups. No statistically significant changes occurred in these parameters at 90 and $180 \mathrm{~min}$ of protocol in both groups.

PVC were observed in one dog of the control group that received RTMCE alone. In this case, PVC were observed immediately after high-mechanical index flash impulses. No PVC or other arrhythmias were observed in dogs that received PESDA plus RTMCE, PESDA alone, or were sham-operated.

\section{Histopathological findings}

No macroscopic abnormalities were observed in the heart or lungs of any animal. Table 1 shows the frequency of microscopi- 
cal lesions obtained by histopathological analysis of the myocardium and lung samples. No significant difference was detected in animals from the PESDA plus RTMCE and control groups.

Mild and focal hemorrhage in the myocardium was similarly observed in both groups (Figure 1). Myocardium contractionbands were observed in one sham-operated dog.

Table 2 shows the mean percentages of 100X microscopical fields presenting microhemorrhages in the myocardium and lung samples. The mean percentages of positive microscopical fields in the myocardium were 0.4 and $0.7 \%$ in the PESDA plus RTMCE and control groups, respectively.

Focal and mild regions of alveolar hemorrhage in pulmonary tissue were observed in the PESDA plus RTMCE and control groups (Figure 2). No significant difference was detected between groups when comparing the hilar and sub-pleural fragments of pulmonary tissue. The mean percentages of positive microscopical fields in the lungs were 2.1 and $1.1 \%$ in the PESDA plus RTMCE and control group, respectively.

\section{Discussion}

In recent years, myocardial contrast echocardiography has been demonstrated to be a feasible and accurate method for the evaluation of myocardial perfusion in many clinical settings (2-5). In addition, the combination of intravenous contrast agents and RTMCE with application of high-mechanical index impulses for microbubble destruction followed by myocardial replenishment has permitted the quantification of myocardial blood flow $(19,20)$. However, some issues still need to be addressed before the widespread use of this technique in clinical practice. Previous studies have demonstrated adverse effects, including microvasculature damage, with contrast agents and ultrasound. Although these studies were restricted to small animals (6-9), they raised concerns regarding the safety of high-mechanical index ultrasound and intravenous contrast agents.

In the present study, we evaluated the bioeffect of a long period of exposure to albumin-encapsulated microbubbles and continuous imaging with the RTMCE modality associated with intermittent high-mechanical index, using a closed-chest canine model. We demonstrated that RTMCE and
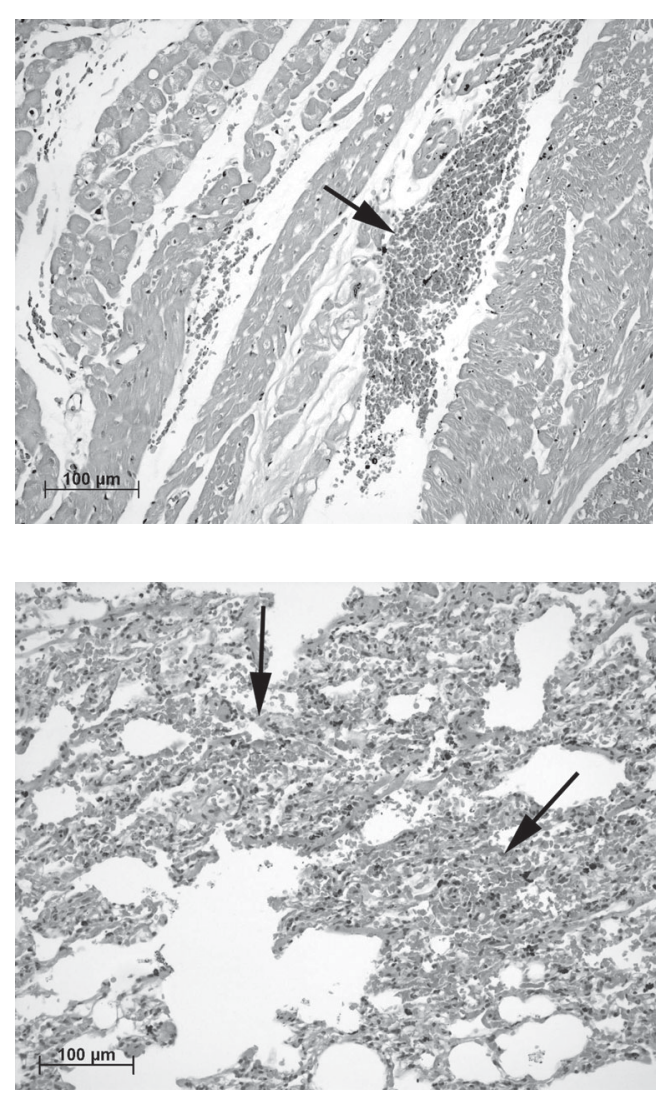

Figure 2. Photomicrography of a sub-pleural lung sample showing mild hemorrhage (arrows), characterized by erythrocytes inside the alveolar spaces. Hematoxylin-eosin; bar $=100 \mu \mathrm{m}$.
Table 2. Mean percentages of $100 \mathrm{X}$ microscopical fields presenting microhemorrhage in myocardium and lung sections.

\begin{tabular}{lcccc}
\hline & $\begin{array}{c}\text { PESDA + RTMCE } \\
(\mathrm{N}=8)\end{array}$ & \multicolumn{3}{c}{ Control group (N = 8) } \\
\cline { 3 - 5 } & & $\begin{array}{c}\text { PESDA } \\
\text { alone }\end{array}$ & $\begin{array}{c}\text { RTMCE } \\
\text { alone }\end{array}$ & $\begin{array}{c}\text { Sham- } \\
\text { operated }\end{array}$ \\
\hline $\begin{array}{l}\text { Myocardium } \\
\text { Lung }\end{array}$ & $0.4 \%$ & $1.3 \%$ & $0.6 \%$ & $0.6 \%$ \\
\hline
\end{tabular}

PESDA = perfluorocarbon-exposed sonicated dextrose albumin; RTMCE = real-time myocardial contrast echocardiography. 
PESDA permitted simultaneous assessment of myocardial perfusion and function with a good safety profile, with no cardiac or pulmonary tissue necrosis as confirmed by pathological analysis. Although some limited areas of focal microhemorrhage were present, they were detected at a similar rate in both the control and PESDA plus RTMCE groups. Pulmonary perivascular microhemorrhage was observed in two dogs from the RTMCE protocol, and myocardial contraction bands were detected in only one of the dogs from the control group (sham-operated). These bands may correspond to recent ischemic myocardial necrosis. However, all these lesions represented minimal tissue involvement when compared to the total area, and could be attributed to the surgical manipulation as well as the trauma resulting from mechanical ventilation.

The effects of microbubble cavitation by ultrasound have been already demonstrated in vitro and in vivo $(12,21,22)$. The microbubbles used in our study (PESDA) consist of decafluorobutane gas surrounded by an albumin shell, with sufficient stability to cross the pulmonary capillary barrier and to reach the left cardiac cavities and coronary microcirculation. Porter and Xie (23) were the first to show the safety profile and utility of intravenously injected PESDA in 28 patients, using intermittent harmonic triggered imaging. The authors demonstrated the possibility of obtaining myocardial perfusion using a small bolus of PESDA at a concentration of $1.4 \times 10^{9}$ microbubbles $/ \mathrm{mL}-0.005$ $\mathrm{mL} / \mathrm{kg}$ in dogs and $0.0025 \mathrm{~mL} / \mathrm{kg}$ in humans - with no significant adverse effects. Other experimental studies have shown no change in cardiac output or in systolic and diastolic pressure in the left ventricle, with a minimal increase in mean systolic pulmonary pressure when using a $0.30 \mathrm{~mL} / \mathrm{kg}$ dose of PESDA (24-26). Mathias Jr. et al. (27) studied 68 patients who underwent dobutamine stress echocardiography using PESDA for enhancement of endocardial border delinea- tion. None of the patients presented serious cardiac arrhythmia or important side effects and no significant changes were observed in blood pressure, respiratory frequency or oxygen saturation.

In the present study, we used the dose of $0.20 \mathrm{~mL} / \mathrm{kg}$ PESDA diluted in $80 \mathrm{~mL}$ normal saline, administered continuously for $180 \mathrm{~min}$ at a rate of $2-4 \mathrm{~mL} / \mathrm{min}$, which is approximately seven times higher than the dose recommended for humans. This high dose and long exposure did not result in any adverse effects in dogs. There is some evidence that the intravenous injection of contrast agents is associated with induction of premature ventricular depolarizations when using high-mechanical index ultrasound. In general, the extension of bioeffects produced by contrast agents is proportional to the pressure amplitude (Mega Pascals) of exposure. Lower frequencies (for example, 1 to $2 \mathrm{MHz}$ ) of exposure are more effective than higher frequencies. One study using intravenous contrast agents has reported an increased number of PVC when applying end-systolic-triggered imaging at a mechanical index of 1.5 (28). The induction of arrhythmias was related to both the dose of the contrast agent and the acoustic pressure studied. In contrasty, Raisinghani et al. (29) reported no increase in the number of PVC in patients being imaged with triggered ultrasound at a high-mechanical index during dipyridamole stress. In our study, we did not observe changes in mean arterial blood pressure, central venous pressure, or heart rate during any of the protocols. PVC were observed in one dog from the control group which did not receive microbubbles. No arrhythmias were observed in the dogs which received PESDA plus RTMCE, even after high-mechanical index flash impulses.

\section{Limitations}

The histopathological analysis performed in our study cannot completely rule out the 
possibility of minor microvascular or endothelial damage, which could only be detected by electron microscopy. On the other hand, even if these lesions occurred, they did not cause significant acute tissue damage, as demonstrated by our results. Tissue sampling was considered adequate, since no macroscopical lesions were detected and because sections were taken from areas most prone to injury due to their topographical location.

The effects of RTMCE and other currently used contrast agents such as lipidencapsulated microbubbles were not tested in the present study. Although the safety profile of commercially available lipid-encapsulated microbubbles has already been demonstrated in humans (30), the bioeffect of such a prolonged exposure to ultrasound has not been previously reported. Further studies are still necessary to address this issue.

In this experimental model, we demonstrated that RTMCE permitted simultaneous evaluation of myocardial perfusion and function, without significant myocardial or pulmonary lesion in the setting of prolonged infusion of PESDA.

\section{References}

1. Wei K, Jayaweera AR, Firoozan S, Linka A, Skyba DM, Kaul S. Quantification of myocardial blood flow with ultrasound-induced destruction of microbubbles administered as a constant venous infusion. Circulation 1998; 97: 473-483.

2. Porter TR, Xie F, Silver M, Kricsfeld D, Oleary E. Real-time perfusion imaging with low mechanical index pulse inversion Doppler imaging. J Am Coll Cardiol 2001; 37: 748-753.

3. Elhendy A, O'Leary EL, Xie F, McGrain AC, Anderson JR, Porter TR. Comparative accuracy of real-time myocardial contrast perfusion imaging and wall motion analysis during dobutamine stress echocardiography for the diagnosis of coronary artery disease. $J$ Am Coll Cardiol 2004; 44: 2185-2191.

4. Tsutsui JM, Xie F, McGrain AC, Mahrous H, Hankins J, O'Leary EL, et al. Comparison of low-mechanical index pulse sequence schemes for detecting myocardial perfusion abnormalities during vasodilator stress echocardiography. Am J Cardiol 2005; 95: 565-570.

5. Dourado PM, Tsutsui JM, Mathias Jr W, Andrade JL, da Luz PL, Chagas AC. Evaluation of stunned and infarcted canine myocardium by real time myocardial contrast echocardiography. Braz $\mathrm{J}$ Med Biol Res 2003; 36: 1501-1509.

6. Dalecki D, Keller BB, Carstensen EL, Neel DS, Palladino JL, Noordergraaf A. Thresholds for premature ventricular contractions in frog hearts exposed to lithotripter fields. Ultrasound Med Biol 1991; 17: 341-346.

7. Dalecki D, Child SZ, Raeman CH, Cox C, Penney DP, Carstensen EL. Age dependence of ultrasonically induced lung hemorrhage in mice. Ultrasound Med Biol 1997; 23: 767-776.

8. Porter TR, Xie F. Therapeutic ultrasound for gene delivery. Echocardiography 2001; 18: 349-353.

9. Price RJ, Skyba DM, Kaul S, Skalak TC. Delivery of colloidal particles and red blood cells to tissue through microvessel ruptures created by targeted microbubble destruction with ultrasound. Circulation 1998; 98: 1264-1267.

10. Chen S, Kroll MH, Shohet RV, Frenkel P, Mayer SA, Grayburn PA. Bioeffects of myocardial contrast microbubble destruction by echocardiography. Echocardiography 2002; 19: 495-500.

11. Unger EC, Hersh E, Vannan M, Matsunaga TO, McCreery T. Local drug and gene delivery through microbubbles. Prog Cardiovasc Dis 2001; 44: 45-54.

12. Ay T, Havaux X, Van Camp G, Campanelli B, Gisellu G, Pasquet A, et al. Destruction of contrast microbubbles by ultrasound: effects on myocardial function, coronary perfusion pressure, and microvascular integrity. Circulation 2001; 104: 461-466.

13. Miller DL, Gies RA. The influence of ultrasound frequency and gasbody composition on the contrast agent-mediated enhancement of vascular bioeffects in mouse intestine. Ultrasound Med Biol 2000; 26: 307-313.

14. MacRobbie AG, Raeman CH, Child SZ, Dalecki D. Thresholds for premature contractions in murine hearts exposed to pulsed ultrasound. Ultrasound Med Biol 1997; 23: 761-765.

15. Becker H, Burns P. Handbook of contrast echocardiography: Left ventricular function and myocardial perfusion. New York: SpringerVerlag Publishers; 2000.

16. Pelberg RA, Wei K, Kamiyama N, Sklenar J, Bin J, Kaul S. Potential advantage of flash echocardiography for digital subtraction of Bmode images acquired during myocardial contrast echocardiography. J Am Soc Echocardiogr 1999; 12: 85-93.

17. Anonymous. Position of the American Heart Association on the use of research animals. A statement for health professionals from a task force appointed by the Board of Directors of the American Heart Association. Circ Res 1995; 57: 330-331.

18. Schiller NB, Shah PM, Crawford M, DeMaria A, Devereux R, Feigenbaum $\mathrm{H}$, et al. Recommendations for quantitation of the left ventricle by two-dimensional echocardiography. American Society of Echocardiography Committee on Standards, Subcommittee on Quantitation of Two-Dimensional Echocardiograms. J Am Soc Echocardiogr 1989; 2: 358-367.

19. Peltier M, Vancraeynest D, Pasquet A, Ay T, Roelants V, D'hondt $\mathrm{AM}$, et al. Assessment of the physiologic significance of coronary disease with dipyridamole real-time myocardial contrast echocardiography. Comparison with technetium-99m sestamibi single-photon emission computed tomography and quantitative coronary angiography. J Am Coll Cardiol 2004; 43: 257-264.

20. Shimoni S, Frangogiannis NG, Aggeli CJ, Shan K, Verani MS, 
Quinones MA, et al. Identification of hibernating myocardium with quantitative intravenous myocardial contrast echocardiography: comparison with dobutamine echocardiography and thallium-201 scintigraphy. Circulation 2003; 107: 538-544.

21. Porter TR, Xie F. Ultrasound, microbubbles, and thrombolysis. Prog Cardiovasc Dis 2001; 44: 101-110.

22. Porter TR, Everbach C, Kricsfeld D, Xie F. Myocardial cavitational activity during continuous infusion and bolus intravenous injections of perfluorocarbon-containing microbubbles. J Am Soc Echocardiogr 2001; 14: 618-625.

23. Porter TR, Xie F. Transient myocardial contrast after initial exposure to diagnostic ultrasound pressures with minute doses of intravenously injected microbubbles. Demonstration and potential mechanisms. Circulation 1995; 92: 2391-2395.

24. Porter TR, Xie F, Kricsfeld D, Armbruster RW. Improved myocardial contrast with second harmonic transient ultrasound response imaging in humans using intravenous perfluorocarbon-exposed sonicated dextrose albumin. J Am Coll Cardiol 1996; 27: 1497-1501.

25. Porter TR, Kricsfeld A, Deligonul U, Xie F. Detection of regional perfusion abnormalities during adenosine stress echocardiography with intravenous perfluorocarbon-exposed sonicated dextrose albu- min. Am Heart J 1996; 132: 41-47.

26. Porter TR, Xie F, Kricsfeld A, Deligonul U, Kilzer K, Kricsfeld D. Myocardial perfusion abnormalities during low-dose dobutamine after coronary reperfusion can be demonstrated with intravenous perfluorocarbon-exposed sonicated dextrose albumin ultrasound contrast. Am Heart J 1996; 131: 1079-1087.

27. Mathias Jr W, Arruda AL, Osório A, Mattos E, Boloneti C, Schwerz $\mathrm{V}$, et al. Improved endocardial border delineation during dobutamine-atropine echocardiography using perfluorocarbon containing microbubbles. J Am Coll Cardiol 1999; 33: 465A.

28. van Der Wouw PA, Brauns AC, Bailey SE, Powers JE, Wilde AA. Premature ventricular contractions during triggered imaging with ultrasound contrast. J Am Soc Echocardiogr 2000; 13: 288-294.

29. Raisinghani A, Wei KS, Crouse L, Villanueva F, Feigenbaum H, Schiller NB, et al. Myocardial contrast echocardiography (MCE) with triggered ultrasound does not cause premature ventricular complexes: evidence from PB127 MCE studies. J Am Soc Echocardiogr 2003; 16: 1037-1042.

30. Tsutsui JM, Elhendy A, Xie F, O'Leary EL, McGrain AC, Porter TR. Safety of dobutamine stress real-time myocardial contrast echocardiography. J Am Coll Cardiol 2005; 45: 1235-1242. 